J. RADIOANAL.NUCL.CHEM., LETTERS $85 / 5 / 285-292 / 1984 /$

PRODUCTION OF ${ }^{232} \mathrm{~Pa}$ FROM THORIUM

G.T. Baldwin*, H.C. Griffin, G.F. Knoll

University of Michigan,
Ann Arbor, Michigan 48109, USA

Received 20 December 1983

Accepted 4 January 1984

A procedure is described for the accelerator production of $232 \mathrm{~Pa}$ and its isolation in radiochemically pure form, suitable for isotope tracing of protactinium/thorium chemical separations.

INTRODUCTION

Activation methods to determine the neutron capture cross section of thorium often involve the measurement of ${ }^{232} \mathrm{~Pa}$, which is present in the radioactive decay sequence

$$
{ }^{232} \mathrm{Th} / \mathrm{n}, \gamma / /^{235} \mathrm{Th} \frac{\beta^{-}}{\mathrm{T}=22 \mathrm{~min}}{ }^{232} \mathrm{~Pa} \frac{\beta^{-}}{\mathrm{T}=27 \mathrm{~d}}{ }^{233} \mathrm{U} .
$$

We have made a measurement ${ }^{1,2}$ in which we chemically separated protactinium from the thorium before measuring the ${ }^{233} \mathrm{~Pa}$ activity. We thereby removed the natural thorium background radiation, and concentrated the trace

\footnotetext{
Present address: Sandia National Laboratories, Division 1232, Albuquerque, NM 87185
} 
BALDWIN et al.: RRODUCTION OF $232 \mathrm{~Pa}$

amount of ${ }^{233} \mathrm{~Pa}$ for $\gamma$-counting. Because some of the protactinium may have been lost during the chemical

separation, it was necessary to measure directly the recovered yield of ${ }^{233} \mathrm{~Pa}$ by tracing the separation process with a known quantity of a different protactinium isotope.

We considered using ${ }^{231} \mathrm{~Pa}$ as the tracer isotope, but rejected it for two reasons. First, ${ }^{231} \mathrm{~Pa}$ is a long-lived $\alpha$ emitter with a radiological hazard similar to that of plutonium, procurement and use of ${ }^{231_{\mathrm{Pa}}}$ is strictly controlled. Second, a particle counting would be necessary, in addition to the $\gamma$-counting needed for ${ }^{233} \mathrm{~Pa}$. The $\gamma$-radiation from ${ }^{231_{\mathrm{Pa}}}$ is too low in energy and intensity to be conveniently measured from bulk samples.

We preferred to use ${ }^{232} \mathrm{~Pa}$, which is readily detected by $\gamma$-counting. The only possible disadvantage to ${ }^{232} \mathrm{~Pa}$ is that it must be produced immediately before use, because it has a half-life of only 1.3 days. ${ }^{232} \mathrm{~Pa}$ could easily be made by neutron activation ${ }^{231} \mathrm{~Pa} / \mathrm{n}, \mathrm{\gamma} /{ }^{232} \mathrm{~Pa}$ : the cross section for activation in a reactor spectrum is about 200 barns. However, this approach would still require the use of ${ }^{231} \mathrm{~Pa}$.

We instead developed a novel method to produce ${ }^{232} \mathrm{~Pa}$ by irradiating thorium with protons ${ }^{232} \mathrm{Th} / \mathrm{p}, \mathrm{n} /{ }^{232} \mathrm{~Pa}$ and isolating radiochemically pure ${ }^{232} \mathrm{~Pa}$ by chemical separation.

\section{PRELIMINARY CALCULATIONS}

A variety of reactions are possible when thorium is bombarded with protons. The cross sections for a few of these reactions have been reported by Tewes ${ }^{2}$ /see Fig. 1./. The ${ }^{232} \mathrm{Th} / \mathrm{p}, \mathrm{n} / \mathrm{I}^{232} \mathrm{~Pa}$ reaction appeared to have an adequately large cross section within the energy range of electrostatic accelerators /e.g., 10-12 MeV/. How- 
BAIDWIN et al.: PRODUCTION OF ${ }^{232} \mathrm{~Pa}$

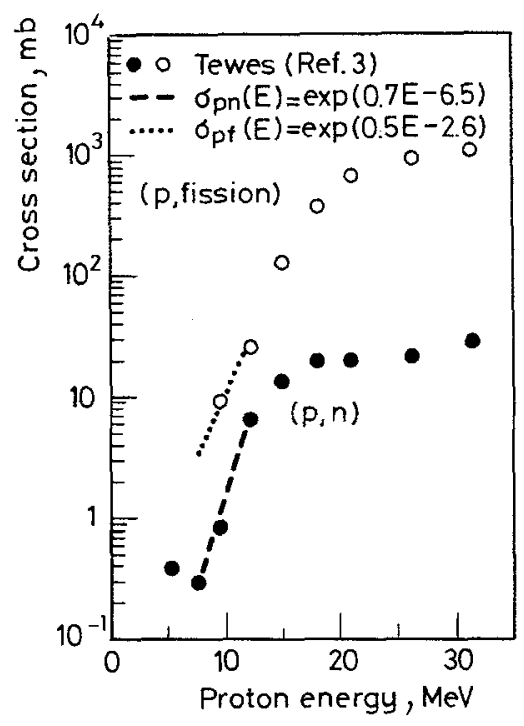

Fig. 1. Cross sections for proton reactions in thorium

ever, the cross section for proton-induced fission is even larger in this energy range, and therefore posed a potential complication.

We made preliminary calculations to determine the preferred irradiation conditions. We interpolated the $/ \mathrm{p}, \mathrm{n} /$ and fission reaction cross section in the energy range of interest from Tewes' data/see Fig. 1./. We assumed the stopping power for protons in thorium given by Anderson and ziegler ${ }^{4}$ in order to obtain the proton energy as a function of depth in the thorium target, for various incident proton energies $/ E_{0} /$. We then averaged each cross section over the target thickness | $\triangle \mathrm{x} \mid$ :

$$
\bar{\sigma} / E_{0}, \Delta x /=\frac{\int_{0}^{\Delta x} \sigma / E / E / x / d x}{\int_{0}^{\Delta x} E / x / d x}
$$


BALDWIN et al.: PRODUCTION OF ${ }^{232} \mathrm{~Pa}$

We found that higher incident beam energies were preferable for two reasons: generation of ${ }^{232} \mathrm{~Pa}$ would be higher /likely nine times greater at $12 \mathrm{MeV}$ than at 9 $\mathrm{MeV} /$, and the fission-to-capture ratio would be lower lat $12 \mathrm{MeV}$, about half of that of $9 \mathrm{MeV} /$. We expected the saturated ${ }^{232} \mathrm{~Pa}$ activity to be about $7 \times 10^{6} \mathrm{~Bq}$ per microampere of incident beam current at $12 \mathrm{MeV}$. Irradiation to saturation was not done, however, due to fission product accumulation and the long exposure time required.

We also estimated the amount of fission product activity and target heating. The expected number of fission events was calculated from the target-average cross section, and the resulting radioactivity was inferred from an empirical formula based on neutron fission of uranium ${ }^{4}$. For a four-hour irradiation at $12 \mathrm{MeV}$ and $1 \mu \mathrm{A}$ beam current, the fission product $\gamma$ activity /mean energy $=700 \mathrm{keV} /$ would be a few times $10^{8} \mathrm{~Bq}$ shortly after the irradiation, decaying to about $15 \%$ of that value after $1 \mathrm{~h}$ and to $2 \%$ after $12 \mathrm{~h}$. Due to high melting point of thorium and the conduction heat sink afforded by the sample holder, target heating was not expected to be a problem.

\section{Target irradiation}

We made arrangements to use the tandem van de Graaff accelerator at Western Michigan University in Kalamazoo, Michigan. Maximum attainable proton energy and beam current were limited by operating conditions to approximately $11 \mathrm{MeV}$ and $0.6 \mu \mathrm{A}$, respectively. The beam spot was about $0.3 \mathrm{~cm}$ in diameter. A bare thorium metal foil, $1.3 \times 10^{-2} \mathrm{~cm}$ thick, was irradiated for $3.5 \mathrm{~h}$. A multichannel analyzer spectrum of the irradiated tar- 


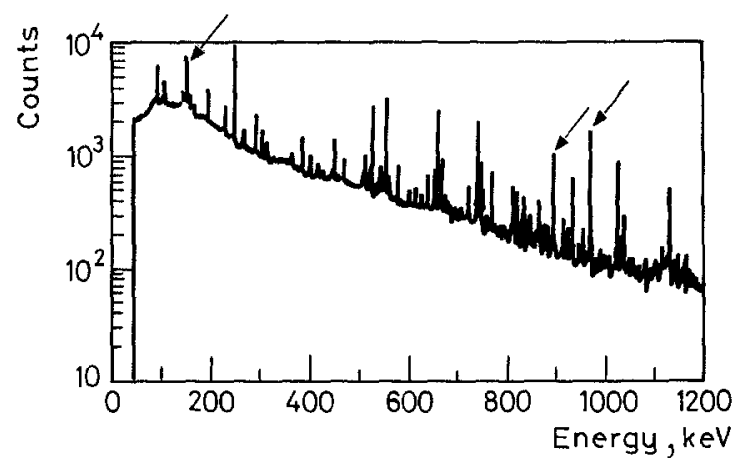

Fig. 2. Gamma spectrum of proton-irradiated thorium larrows point to $232 \mathrm{~Pa}$ lines at 150,894 and $969 \mathrm{keV} /$

get foil was acquired about $7 \mathrm{~h}$ after the end of irradiation, using a Ge/Li/detector /see Fig. 2./. The large number of $\gamma$-lines in Fig. 2. is due mainly to fission products and natural thorium decay chain isotopes. ${ }^{232} \mathrm{~Pa}$ is also present, as evident by the stronger ${ }^{232} \mathrm{~Pa}$ lines at 150, 894 and $969 \mathrm{keV} /$ indicated by arrows in Fig. 2.1. The total amount of ${ }^{232} \mathrm{pa}$ present in the sample at the end of the irradiation was estimated to be roughly $1-2 \times 10^{7} \mathrm{~Bq}$.

Isolation of ${ }^{232} \mathrm{~Pa}$

The thorium foil probably weighed less than $0.5 \mathrm{~g}$ after being trimmed to remove excess metal loutside of the faintly visible beam spot/. The sample was slowly dissolved with $10 \mathrm{ml} 6 \mathrm{M} \mathrm{HCl}$ to which 4 drops of $1 \mathrm{M} \mathrm{HF}$ had been added to aid in the dissolution. The fluoride ion was then complexed by the addition of $0.24 \mathrm{~g}$ $\mathrm{AlCl}_{3} \cdot 6 \mathrm{H}_{2} \mathrm{O}$. The separation was done by solvent extraction, using a procedure adapted from the work of Moore and Reynolds ${ }^{6}$ and Scherff and Herrmann ${ }^{7}$. Because of the small quantities involved, the extractions were 
BALDWIN et al.: PRODUCTION OF $232 \mathrm{~Pa}$

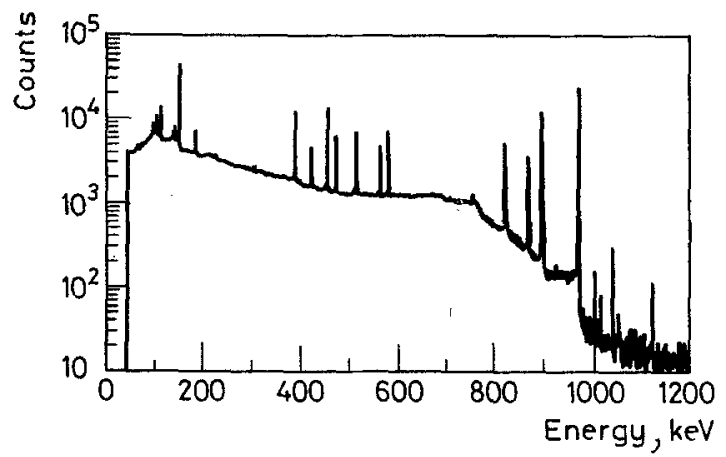

Fig. 3. Gamma spectrum of ${ }^{232} \mathrm{~Pa}$ separated from protonirradiated thorium

carried out in centrifuge tubes. Mixing was done with the aid of a vortex agitator and the separation of phases was done by centrifuging. Phases were removed by capillary pipette.

protactinium in the target solution was extracted into $8 \mathrm{ml}$ undiluted disobutylcarbinol /DIBC/. The DIBC was washed twice with $8 \mathrm{ml} 6 \mathrm{M} \mathrm{HCl}$, and then twice more with $8 \mathrm{ml} 6 \mathrm{M} \mathrm{HCl} / 4 \%$ oxalic acid. Next, the protactinium was stripped from the DIBC three times in succession, each using $1 \mathrm{ml} 8 \%$ oxalic acid. The three strip solutions were combined, adjusted to extractable form by adding $3 \mathrm{ml} 12 \mathrm{M} \mathrm{HCl}$, and reextracted into $3 \mathrm{ml} \mathrm{DIBC}$. The DIBC was then stripped once with $1 \mathrm{ml} 2 \mathrm{M} \mathrm{HCl}$ containing 1 drop $1 \mathrm{M} \mathrm{HF}$.

The $1 \mathrm{ml} \mathrm{HCl} / \mathrm{HF}$ solution containing protactinium was then transferred to a small snap-cap polyethylene vial and analyzed with a Ge/Li/ detector. Fig. 3. is the $\gamma$-spectrum obtained. Excellent radiochemical purity was achieved: with the exception of the annihilation peak at $511 \mathrm{keV}$ and one line at $1039 \mathrm{keV} /$ probably ${ }^{66} \mathrm{Cu} /$, all of the peaks visible in Fig. 3. are from ${ }^{232} \mathrm{~Pa}$. 
BALDWIN et al.: PRODUCTION OF ${ }^{232} \mathrm{~Pa}$

We very much appreciate helpful assistance from L.D. Oppliger and the Physics Department at Western Michigan University. This work has been supported by the U.S. Department of Energy under contract number C-EY-78-S-O2-2025.

REFERENCES

1. G.T. Baldwin, PhD Dissertation, University of Michigan, 1981 .

2. G.T. Baldwin, G.F. Knoll, "Absolute Measurement of the Cross-Section for $23-\mathrm{keV}$ Neutron Activation of Thorium". Nucl. Sci. Eng./to be published/.

3. H.A. Tewes, Phys. Rev., $98 / 1955 / 25$.

4. H.H. Anderson, J.F. Ziegler, Hydrogen Stopping Powers and Ranges in AII Elements, NY: Pergamon Press, 1977.

5. J.R. Lamarsh, Introduction to Nuclear Engineering, Addison-Wesley, 1975.

6. F.L. Moore, S.A. Reynolds, Anal. Chem., 29 /1957/ 1596 .

7. H.L. Scherff, G. Herrmann, Radiochim. Acta, 8 /1966/ 53. 